\title{
Mathieu Potte-Bonneville, Recommencer, Lagrasse, Verdier, 2018, 80 p.
}

\section{Rudolf Mahrer}

\section{(2) OpenEdition}

1 Journals

\section{Édition électronique}

URL : http://journals.openedition.org/genesis/3249

DOI : 10.4000/genesis.3249

ISSN : 2268-1590

Éditeur :

Presses universitaires de Paris Sorbonne (PUPS), Société internationale de génétique artistique littéraire et scientifique (SIGALES)

Édition imprimée

Date de publication : 15 décembre 2018

Pagination : 181

ISBN : 979-10-231-06282

ISSN : 1167-5101

\section{Référence électronique}

Rudolf Mahrer, « Mathieu Potte-Bonneville, Recommencer, Lagrasse, Verdier, 2018, 80 p. », Genesis [En ligne], 47 | 2018, mis en ligne le 15 janvier 2019, consulté le 21 janvier 2021. URL : http:// journals.openedition.org/genesis/3249; DOI : https://doi.org/10.4000/genesis.3249

Ce document a été généré automatiquement le 21 janvier 2021.

Tous droits réservés 


\title{
Mathieu Potte-Bonneville, Recommencer, Lagrasse, Verdier, 2018, 80 p.
}

\author{
Rudolf Mahrer
}

\section{RÉFÉRENCE}

Mathieu Potte-Bonneville, Recommencer, Lagrasse, Verdier, 2018, 80 p.

1 «La moindre remarque marginale fait que si je relis jamais ce livre, je reprends le fil de mes idées et vais en avant. Si je ne trouve aucun souvenir en relisant un livre, le travail est à recommencer ${ }^{1}$." Pour " aller de l'avant ", Stendhal annote ses livres au fil de la lecture. L'écrit ajouté à l'écrit assiste sa mémoire et fixe son expérience du texte. Le livre porte par suite les traces de l'espoir que la prochaine lecture ajoute au sens produit par la première. Qu'il y ait continuation du geste interprétatif plutôt que répétition. Stendhal ne veut pas recommencer.

Commencer, continuer, finir, recommencer: ces «flexions» de l'agir humain concernent au premier chef le créateur, qui doit savoir les décliner, s'il veut faire œuvre. C'est pour cela que le généticien des productions culturelles trouve dans Recommencer de Mathieu Potte-Bonneville une mine de réflexions pertinentes pour penser l'action créative. L'essai concentre en soixante-dix pages, élégantes et parfois poétiques, philosophie, psychanalyse et littérature, parcourant sept motifs du recommencement, qui forment un paradigme familier de la critique génétique : reprise, renaissance, balbutiements, continuation, répétition, ressac et récidive.

Mathieu Potte-Bonneville commence par un saisissant récit : celui de l'effet que produit sur Gottlob Frege, venant de publier ses Lois fondamentales de l'arithmétique, la lettre de Bertrand Russel, qui poursuit les mêmes buts que son aîné. Les paradoxes relevés par le jeune logicien conduiront Frege à considérer que « le travail de sa vie à cette époque 
avait été une erreur » (p. 12). Frege ne s'en remettra pas, Russel consacrera sa vie à s'y remettre. «Comment s'y reprend-on?» (p. 17).

On comprend, dans la deuxième section («Renaissance »), que l'horizon de la réflexion de Potte-Bonneville est d'abord politique: «l'actualité suggère de se tourner vers la politique : y prévaut plus qu'ailleurs l'impression que quelque chose est aujourd'hui à refaire (ce qui implique de reconnaître que quelque chose s'est défait et de prendre la mesure des défaites)»( (p.23). Mais si le philosophe puise dans la littérature (notamment chez Pavese, Beckett et Flaubert) des figures de sujets faisant face aux affres du recommencement, c'est que la question habite nécessairement l'écrivain. Les reprises de l'écriture sont elles aussi motivées par le sentiment d'une défaite ou du moins par la reconnaissance que quelque chose est à refaire. Et les méthodes scripturales, comme les annotations de Stendhal, gagnent évidemment à être considérées dans le cadre général d'une pensée, logique et affective, de ce que commencer, continuer, finir et recommencer veulent dire pour tel écrivain.

Si l'angoisse du retour à zéro oriente l'action, celle-ci peut également être conduite par la nostalgie du retour à l'origine, par « l'éloge du commencement pur, de la limpidité des aubes » (p. 29), par l'élan « vers cette première fois qui ne reviendra pas » (p. 22). La page blanche, matin de toutes les créations possibles, est sans doute une expérience vertigineuse; mais lorsqu'on sait, a posteriori, qu'elle fut l'origine d'un accomplissement, elle devient désirable, comme «l'idée que l'enfance serait poétique ne nous vient qu'à l'âge mûr » (p. 35).

6 Pour Bonne-Potteville, le journal de Cesare Pavese, Le Métier de vivre, témoigne que ces deux pulsions contradictoires (dépasser les débuts / en rêver) étranglaient l'écrivain italien. "Ainsi lit-on, au 1er octobre 1935 : "Une seule chose parmi beaucoup d'autres, me semble insupportable pour l'artiste: ne plus se sentir au début." Mais le jour de Noël 1937 : "Il y a quelque chose de plus triste que vieillir, et c'est de rester enfant." Les deux formules sont rigoureusement contradictoires; mais c'est que la première exprime l'inquiétude de ne plus débuter dans la poésie [...]; cependant que l'autre exprime l'angoisse de ne jamais faire, dans la vie, que revenir au début » (p. 33-34).

7 Sur le plan conceptuel, recommencer, c'est inscrire une action singulière dans une suite d'actions antérieures, mais une suite qui conserve sa valeur inaugurale : «tout entier inauguration, et tout entier rappel»(p. 22). Recommencer, c'est donc d'une certaine façon continuer. Mais d'une certaine façon seulement, car recommencer "s'adosse souvent au constat exaspéré que "ça ne peut pas continuer" » (p. 37). Pour repartir en effet, "il faut tirer un trait, sans qu'on sache s'il s'agit de tracer ou de biffer, de marquer une frontière ou une rature - à moins que ce ne soit au fond la même chose tant la réalité d'une frontière s'authentifie de désigner ceux qui ne la passeront pas " (p. 38). Le sentiment du recommencement contribue ainsi à structurer nos actions, à leur donner un contour. Recommencer « introduit une différence significative dans le flux ininterrompu des événements et une démarcation dans ma propre mémoire " (p. 38). Ce flux, magmatique et informe, le philosophe l'appelle le « continuel » (p. 39). À la figure de Pavese, comme « persistance de l'inchoatif » répond ici celle de Beckett qui, dans L'Innommable, En attendant Godot ou Pour en finir encore, donne voix à cette hantise de la durée où rien n'arrive, comme une narration sans intrigue, où le sujet devient luimême indistinct, faute de prendre corps dans l'expérience. S'ensuit une «rage de terminer » qui prend la forme d'une marche vers l'horizon. Ces considérations sur le continuel éclaire d'un jour inattendu cette citation de Beckett qu'arborent plusieurs 
sportifs contemporains : « Ever tried. Ever failed. No matter. Try again. Fail again. Fail better. " Il s'agirait non pas de "surmonter l'échec par la répétition, mais bien la répétition par l'échec » (p. 45), échapper au continuel, quoi qu'il en coûte.

"Répétition » (cinquième section) situe la réflexion sur nos actions entre l'angoisse de ne pas en voir le bout et celle d'être toujours ramené au même point. Sous cet angle, recommencer c'est cette fois répéter; pourtant il n'y a pas de « recommencement qui ne se dresse contre le retour à l'identique que la répétition suppose» (p. 50-51). C'est le rapport au savoir qu'interroge ce moment de la réflexion : recommencer, «fraîcheur instruite » (p. 50), consiste à espérer que quelque chose de la première fois fasse retour, mais aussi que l'expérience acquise permette à la reprise d'aller plus loin. "Savoir mieux, vouloir davantage» (p. 48) s'annonce comme les prémisses de la reprise. En s'appuyant d'abord sur Lacan et la figure du "sujet supposé savoir ", que représente l'analyste, et sa nécessaire chute dans l'élucidation du désir et l'émancipation de l'analysant, Potte-Bonneville invite plutôt à considérer comme une illusion le pouvoir de la connaissance sur le succès de l'entreprise recommencée. Avec Hans Bloomenberg ${ }^{2}$ ensuite, l'essayiste envisage le rôle de la rétrospection dans le processus du recommencement : pourquoi se donner des précurseurs, c'est-à-dire représenter son action comme le recommencement d'une action ancienne? Bloomenberg, qui aborde cette problématique par la notion de préfiguration, considère «la répétition comme ressource et comme piège ; il en fait la condition d'une action soustraite à l'arbitraire subjectif, et une matrice de délire où le sujet peut rêver être tous les noms de l'histoire» (p.52). Voilà qui incite à se demander comment la préfiguration conditionne l'écriture, si quelque chose caractériserait la genèse d'une œuvre posée, in statu nascendi, dans le sillage d'une œuvre antérieure à recommencer.

9 «Ressac » envisage la langue comme le modèle même du recommencement, dans une argumentation familière aux linguistiques de l'énonciation qui ont inspiré les généticiens : « parler est pour chacun cet événement dans lequel l'enjeu et l'inquiétude sont de recommencer, c'est-à-dire de ne pouvoir initier d'expression que dans la répétition d'une langue d'avant nous, par elle et malgré elle»(p.57). Dès lors, l'énonciation au sens de Benveniste (que ne mentionne pas Potte-Bonneville) constitue non pas une figure parmi d'autres du recommencement, mais l'articulation elle-même du singulier et du général, du subjectif et du social, et le philosophe d'en conclure que c'est à la littérature que revient de construire dans le langage « les figures médiatrices et poétiques qui nous aideraient à repartir »: " parce que l'enjeu est, au fond, de savoir comment l'on cesse d'être sujet au recommencement (comme on l'est aux allergies, au rhume, aux phobies), pour devenir sujet $d u$ recommencement, il vaudrait la peine de scruter le double mouvement par lequel, d'un même pas, les narrations contemporaines relancent la langue, dressent des profils de subjectivité comme on jette des ponts, inventent des personnages qui aux petites heures viennent et reviennent nous fréquenter» (p. 63-64). Le petit volume se referme ("Récidive») d'ailleurs sur la figure du recommencement que propose Flaubert avec les deux copistes Bouvard et Pécuchet : d'où vient à ces deux-là leur « aptitude si increvable à récidiver » (p. 71) ? De leur bêtise, qui signifierait « la relation des savoirs objectifs aux sujets connaissants, en tant que ce rapport échoue radicalement à transformer en quoi que ce soit les uns ou les autres"(p.69). Ainsi leur savoir de copie, sans effet et vain, conduit à un recommencement perpétuel - qui finalement trahit une certaine force et tient les procrastinateurs en respect. À ce titre, Bouvard et Pécuchet enrichissent le répertoire des sujets nouveaux que la littérature nous permet de penser, pour recommencer et 
nous recommencer : «Les écrivains ont plus d'un tour pour vous remettre le pied à l'étrier» (p. 72, clausule).

10 Au final, la lecture du petit livre de Potte-Bonneville rappellera, au besoin, que pour décrire l'écriture, une théorie de l'action est nécessaire. Non seulement en tant que modèle théorique auquel rapporter les procès d'écriture, mais aussi en tant que modèle « embarqué » par les scripteurs eux-mêmes et guidant leur action.

\section{NOTES}

1. Stendhal, Journal, 31 juillet 1804, cité par Jean-Louis Lebrave, "Hypertexte - Mémoires Écriture ", Genesis, n 5, 1994, p. 18-24, je souligne.

2. Préfiguration. Quand le mythe fait l'histoire, trad. J.-L. Schlegel, Paris, Le Seuil, 2016.

\section{AUTEURS}

\section{RUDOLF MAHRER}

RUDOLF MAHRER est maître d'enseignement et de recherche à l'Université de Lausanne et corédacteur en chef de la revue Genesis. Ses travaux s'inscrivent dans les traditions de la linguistique du texte et de la linguistique de l'énonciation et accordent une attention particulière aux différences entre oralité et écriture. Il travaille actuellement au développement de la collection numérique « Variance », consacrée à la publication des œuvres ayant été réécrites après publication (variance.ch).

rudolf.mahrer@unil.ch 